\title{
Expression and therapeutic significance of estrogen receptor $\beta$ in malignant pleural mesothelioma
}

First draft submitted: 16 January 2017; Accepted for publication: 19 January 2017; Published online: 17 February 2017

Keywords: estrogen receptor $\beta \bullet$ malignant pleural mesothelioma $\bullet$ prognosis $\bullet$ therapy

Malignant pleural mesothelioma (MPM) is an asbestos-associated aggressive cancer that arises from the mesothelial lining of the pleura. Although the use of asbestos has been banned in many countries, the potentially hazardous exposure to asbestos is still present with locally high incidences of mesothelioma [1]. Chemotherapy for MPM has remained unchanged since 2003 [2], when the combination of cisplatin and pemetrexed became the standard firstline therapy for patients who are not suitable for aggressive surgery, or in whom chemotherapy is recommended as part of a multimodality regimen. Unfortunately, the disease recurs in over $80 \%$ of the patients at the 2 -year followup mark. In the second-line setting, a variety of cytotoxic agents have been tested, either as single or combined treatment [3], but none of these have been adopted as standard therapy. Advances in the understanding of MPM biology bring, however, hope that novel agents will be identified that improve patient outcomes.

The observation that women diagnosed with MPM respond better to treatments and live longer than men, prompted us to assess the role of estrogens and their receptors in this cancer.

Estrogens play important roles in several physiological and pathological processes. The existence of an estrogen receptor (ER) was demonstrated in 1958 [4] and the corresponding gene was cloned in 1985. It was considered the only receptor responsible for mediating estrogen action until the gene cod- ing for a second ER subtype, ER $\beta$, was cloned in 1996 [5]. This second receptor is expressed in many human tissues, including some that traditionally are considered non target for estrogens. One of the more interesting observations about ER $\beta$ is that it exerts antiproliferative effects in different preclinical in vitro and in vivo models of human cancers [6-8].

We analyzed the expression of the two ER subtypes, $\alpha$ and $\beta$, in tumor biopsies from MPM patients and in normal pleura from healthy controls [9]. Nuclear ER $\beta$ immunoreactivity was detected in normal pleura and in the majority of the 78 analyzed MPM samples, although with reduced presence and intensity, compared with normal pleura. The cumulative probability of survival after 2 years of follow-up was $80 \%$ for subjects with high ER $\beta$ expression versus $31 \%$ for subjects with negative of low ER $\beta$ expression ( $p=0.02, \log$-rank test).

Importantly, multivariate analysis of overall survival demonstrated the prognostic significance of ER $\beta$ staining. Different from other lung cancers, none of 78 MPM biopsies or normal pleura stained positive for ER $\alpha$. MPM cells represent, therefore, a powerful model to study the ER $\beta$ role and function.

\section{Key findings}

ER $\beta$ inhibits MPM cell proliferation

We hypothesized that the observed expression of ER $\beta$ in MPM samples and longer survival of MPM patients could reflect its tumor-suppressor properties. To test this

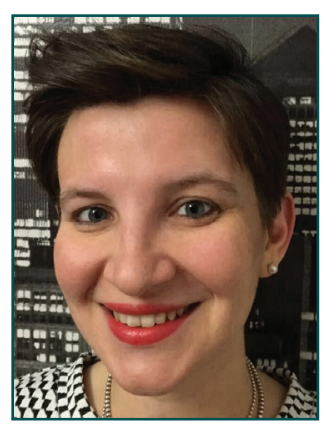

\section{Giulia Pinton}

Department of Pharmaceutical Sciences, University of Piemonte Orientale,Novara, Italy

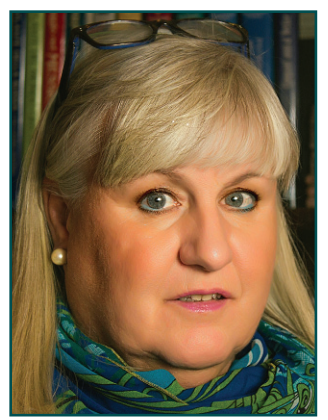

Laura Moro

Department of Pharmaceutical Sciences, University of Piemonte Orientale,Novara, Italy

Tel.: +390321375820

laura.moro@uniupo.it 
hypothesis, we performed studies in vitro providing evidence that ER $\beta$ plays a role in the control of MPM cell proliferation by downregulation of cyclin B1 and survivin, causing a G2-M-phase arrest in cell cycle progression [8]. Then, we performed a meta-analysis of global gene expression profiles of 93 MPM samples to identify an ESR2 (ER $\beta$-coding gene) signature.

Among genes downregulated in tumors expressing high levels of ESR2 we identified $S D H B$, which encodes the B subunit of the succinate dehydrogenase (complex II of the mitochondrial respiratory chain). We demonstrated that ER $\beta$, activated by overexpression or by selective agonist stimulation, impaired complex II and IV activity compromising mitochondrial oxidative phosphorylation. This led to reduced mitochondrial ATP production, increased glycolysis dependence and decreased cell proliferation.

Furthermore, the reported in vitro effects on the mitochondrial respiratory chain complexes translated to an in vivo mesothelioma tumor model treated with the ER $\beta$-selective agonist KB9520 [10].

\section{ER $\beta$ affects epithelial-mesenchymal transition in MPM}

MPM may also be a powerful model for studying epithelial-mesenchymal transition (EMT) in vivo [11]. Aside from the location and stage of the disease, mesothelioma is also categorized by cell type. According to the WHO classification, MPM is subclassified as epithelioid (mostly composed of epithelial-shaped cells), sarcomatoid (mostly composed of spindle-shaped cells) or biphasic (composed of both cell types). The morphological patterns of MPM are therefore likely to be the outcome of different phases in the EMT process. Each cell type responds differently to treatment and has an important effect on a patient's prognosis. Mesothelioma tumors made up of epithelioid cells are the most treatable and patients with this cell type have the best prognosis.

We demonstrated that re-expression of ER $\beta$ in ER-negative cells originating from biphasic MPM conferred a more epithelioid phenotype, decreased capacity for anchorage-independent growth and down-modulated proliferative signal transduction pathways. Conversely, ER $\beta$ knockdown in ER-positive cells conferred a more invasive phenotype, increased anchorage independent growth and elevated EGFR-coupled signal transduction pathways [12].

The possibility to reverse the more aggressive biphasic mesothelioma histotype by targeting ER $\beta$ with a selective agonist could, therefore, represent a novel and important treatment strategy to stabilize this aggressive disease, with manageable toxicity.
ER $\beta$ is expressed by ER-negative MPM cells in hypoxia

Hypoxia is a common feature in MPM. A pilot study performed with [F-18] fluoromisonidazole PET-CT analysis has provided evidence of significant areas of hypoxia in MPM-dominant tumor masses [13]. Another study has described carbonic anhydrase IX positivity, proposed to serve as a surrogate marker of hypoxia, to be predominant in epithelioid MPMs, while not expressed in sarcomatoid and sarcomatoid areas of biphasic MPMs [14]. Differently from other tumors in which the hypoxic condition induces EMT and invasion, we have described that hypoxia causes, in cells derived from biphasic MPM, the switch from spindle to epithelioid phenotype with increased E-cadherin expression and reduced growth rate [15]. Changes in epigenetic marks, including lysine methylation of histones, have been observed in development and in disease states where hypoxia is known to be an important feature. Histone-lysine methylation is dynamically regulated by histone methyltransferases and histone demethylases (KDMs). We reported a strong positive correlation between the expression of the KDM6B and ER $\beta$ in MPM tumors and cell lines. We described that, in hypoxia, the HIF $2 \alpha-$ KDM6B axis induced an epithelioid morphology and ER $\beta$ expression in biphasic MPM cells with ER-negative phenotype. ER $\beta$ was also transiently expressed by ER-negative cells cultured as spheroids in vitro or as tumor mass in vivo when hypoxic conditions occurred. Importantly, ER $\beta$ expression and tumorsuppressive function were maintained by selective ligand activation [16].

\section{ER $\beta$ activation increases sensitivity of MPM to the standard of care}

We investigated the possibility of an additive or synergistic effect between the ER $\beta$ selective agonist KB9520 and the standard of care (cisplatin/pemetrexed) for treatment of MPM. We showed that KB9520 acted as a chemosensitizer through activation of ER $\beta$, increasing the antitumorigenic efficacy of cisplatin or the cisplatin/pemetrexed combination. Treatment with KB9520 in combination with cisplatin/pemetrexed in vivo had greater efficacy than either treatment alone and caused a significantly reduced tumor burden compared with vehicle-treated animals [17]. Importantly, KB9520 had no cytotoxic effect per se and reduced cisplatin toxicity in ER $\beta$-expressing non malignant mesothelial cells. Thus, KB9520 may increase the sensitivity of MPM tumors to the standard of care in patients and perhaps result in higher response rates, without adding toxicity. 


\section{Implications \& future perspective}

Drugs that selectively target ER $\beta$, being free from the undesired ER $\alpha$-promoted proliferative effects on breast and uterus, might be safer than non selective estrogens [18]. Several synthetic and natural ER $\beta$-selective agonists have been identified that have exhibited promising antitumorigenic activity in preclinical cancer models. Another important observation is that ER $\beta$ selective agonists can increase the expression of ER $\beta$ in cells where its expression has been downregulated. Therefore, drugs with selectivity for ER $\beta$ might prove promising in the development of novel, targeted therapies for the clinical management of human cancers.

\section{References}

1 Frank AL, Joshi TK. The global spread of asbestos. Ann. Glob. Health. 80(4), 257-262 (2014).

2 Vogelzang NJ, Rusthoven JJ, Symanowski J et al. Phase III study of pemetrexed in combination with cisplatin versus cisplatin alone in patients with malignant pleural mesothelioma. J. Clin. Oncol. 21, 2636-2644 (2003).

3 Buikhuisen WA, Hiddinga BI, Baas P et al. Second line therapy in malignant pleural mesothelioma: a systematic review. Lung Cancer 89(3), 223-231 (2015).

4 Jacobson HI, Gupta GN, Fernandez C et al. Determination of tritium in biological material. Arch. Biochem. Biophys. 86, 89-93 (1960).

5 Kuiper GG, Enmark E, Pelto-Huikko M et al. Cloning of a novel receptor expressed in rat prostate and ovary. Proc. Natl Acad. Sci. USA 93(12), 5925-5930 (1996).

6 Yakimchuk K, Iravani M, Hasni MS et al. Effect of ligandactivated estrogen receptor $\beta$ on lymphoma growth in vitro and in vivo. Leukemia 25, 1103-1110 (2011).

7 Marzioni M, Torrice A, Saccomanno S et al. An oestrogen receptor $\beta$-selective agonist exerts anti-neoplastic effects in experimental intrahepatic cholangiocarcinoma. Dig. Liver Dis. 44, 134-142 (2012).

8 Jia M, Dahlman-Wright K, Gustafsson JA. Estrogen receptor alpha and beta in health and disease. Best Pract. Res. Clin. Endocrinol. Metab. 29(4), 557-568 (2015).

9 Pinton G, Brunelli E, Murer B et al. Estrogen receptor $\beta$ affects the prognosis of human malignant mesothelioma. Cancer Res. 69(11), 4598-4604 (2009).

10 Manente AG, Valenti D, Pinton G et al. Estrogen receptor $\beta$ activation impairs mesothelioma cell metabolism and growth in vitro and in vivo. Oncogenesis 2, e72 (2013).

\section{Financial \& competing interests disclosure}

The authors have no relevant affiliations or financial involvement with any organization or entity with a financial interest in or financial conflict with the subject matter or materials discussed in the manuscript. This includes employment, consultancies, honoraria, stock ownership or options, expert testimony, grants or patents received or pending, or royalties.

No writing assistance was utilized in the production of this manuscript.

\section{Open access}

This work is licensed under the Creative Commons Attribution 4.0 License. To view a copy of this license, visit http://creativecommons.org/licenses/by/4.0/

11 Fassina A, Cappellesso R, Guzzardo V et al. Epithelialmesenchymal transition in malignant mesothelioma. Mod. Pathol. 25, 86-99 (2012).

12 Pinton G, Thomas W, Bellini P et al. Estrogen receptor $\beta$ exerts tumor repressive functions in human malignant pleural mesothelioma via EGFR inactivation and affects response to gefitinib. PLoS ONE 5(11), e14110 (2010).

13 Francis RJ, Segard T, Morandeau L et al. Characterization of hypoxia in malignant pleural mesothelioma with FMISO PET-CT. Lung Cancer 90 (1), 55-60 (2015).

14 Capkova L, Koubkova L, Kodet R. Expression of carbonic anhydrase IX (CAIX) in malignant mesothelioma. An immunohistochemical and immunocytochemical study. Neoplasma 61(2), 161-169 (2014).

15 Manente AG, Pinton G, Zonca S et al. Intracellular lactate-mediated induction of estrogen receptor beta $(\mathrm{ER} \beta)$ in biphasic malignant pleural mesothelioma cells. Oncotarget 6(28), 25121-25134 (2015).

16 Manente AG, Pinton G, Zonca S et al. KDM6B histone demethylase is an epigenetic regulator of estrogen receptor $\beta$ expression in human pleural mesothelioma. Epigenomics 8(9), 1227-1238 (2016).

17 Pinton G, Manente AG, Daga A et al. Agonist activation of estrogen receptor beta $(\mathrm{ER} \beta)$ sensitizes malignant pleural mesothelioma cells to cisplatin cytotoxicity. Mol. Cancer 13, 227 (2014).

18 Warner M, Huang B, Gustafsson JA. Estrogen Receptor $\beta$ as a pharmaceutical target. Trends Pharmacol. Sci. 38(1), 92-99 (2017). 